\title{
A Tracking Platform Solution for Autonomous Vehicles Localization in Future Smart Cities Using Machine and Deep Learning
}

\author{
Uchenna Charles ONYEMA ${ }^{\mathrm{a}, 1}$, Mahmoud SHAFIK ${ }^{\mathrm{a}, 2}$, \\ Todor DOBREV ${ }^{\mathrm{a}}$ and James HARDY ${ }^{\mathrm{a}}$ \\ ${ }^{a}$ University of Derby, Derby, DE221 GB, UK
}

\begin{abstract}
The localization of autonomous vehicles requires, accurate tracking of its position and orientation in all conditions. As modern cities evolve localization would require a more precise accuracy that up to the level of centimetre and decimetre. One of the most crucial struggles in global positioning system and inertial navigation fusion is that the accuracy of the algorithm is reduced during GPS interruptions. In recent days bigdata, machine and deep learning offer great opportunities, especially for future smart and industrial 4.0 autonomous applications. This research programme is aiming to investigate and deploy machine and deep learning approach to improve and reach the level of reliability, accuracy and robustness required at low-cost GPS/IMU unit. The programme will also present a tracking platform solution that would compensates the issues of lack of accuracy in existing localization methods. The initial result of this ongoing programme is presented and reported in this paper. The paper also covers the research programme future development plans and milestones.
\end{abstract}

Keywords. Autonomous Vehicles, Localization, Kalman Filter, Linearization, Machine and Deep Learning

\section{Introduction}

Localization is one of the five key functional systems which autonomous driving is to achieve. The other being Perception, planning, Control and System Management. Precise Localization of the smart car in a dynamically changing environment is very crucial because marginal to large deviations from actual position could lead to vehicle not tracing appropriate navigation path or failure in vehicle control.

There are a number of sensor-based techniques [2][3] employed in vehicle localization: this includes: The Camera based technique, Radar based technique, Lidar technique, ultrasonic based technique and GPS/IMU technique. Traditional method of carrying out vehicle localization is through Global navigation satellite system (GNSS), which can be the GPS, GLONASS, Galileo etc., providing localization parameter for the vehicle. However, GPS suffer from setbacks such as signal degradation due to high rise buildings,

\footnotetext{
${ }^{1}$ Corresponding Author. u.onyema@derby.ac.uk

${ }^{2}$ Mahmoud Shafik.mshafik@derby.ac.uk
} 
bridges, trees and tunnels, precision issues as and also Time to Fix first (TTFF) [4]. MEMS based Inertial measurement units on the other hand is not without its own defect, as it suffers from drift which is a result of continuous integration. [5][6]

The technology integrates the inertial measurement system with Global positioning systems, have in recent times provided the basis for autonomous vehicle navigation. However, its characteristic low performance continues to be the subject of research to replace and or compensate this available technology. Machine learning algorithms would be explored in this work to enhance performance of the localization module by providing intelligent estimates of Pose differences during GPS loss times based on previously learnt relationships in GPS/INS integration filter, for optimal tracking of vehicle position and attitude.

\section{Global positioning system/Inertial navigation system integration.}

The performance issues, because of GPS and INS sensors defects, increases the need for long term solutions that would minimize positioning error, while providing reliability over time. Integration of the GPS/INS over a Kalman filter provides optimal and more stable estimates of navigation solutions for vehicle localization over time.

\subsection{Kalman filter}

The Kalman filter is used in both static and dynamic system for state estimation. Introduced by Rudolph Kalman in 1960 [7], Its estimation is based on Gaussian probability density function (PDF), which has mean $\overline{X_{k}}$ and covariance P. There are two main stages in the Kalman filtering. The prediction and update stage. The prediction stage is based on a dynamic model of the system, then the obtained state of prediction is updated or corrected based on measurement process.

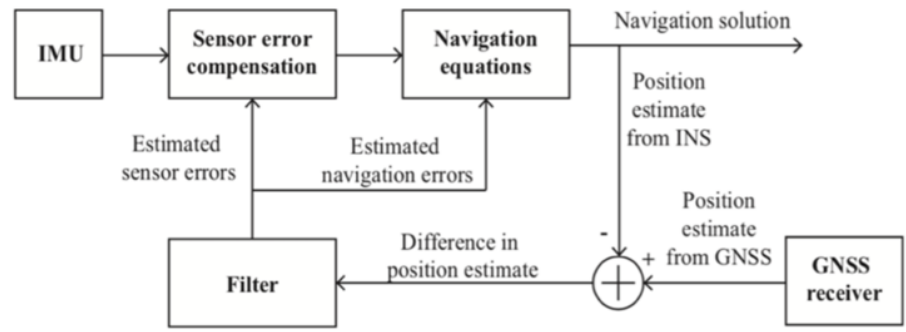

Figure 1: Loosely coupled Kalman filter

The System equations for a discrete time Kalman filter are given below:

\section{A. Time Update:}

Predicted State: $\hat{X}_{k \mid k-1}=F_{k} \hat{X}_{k-1 \mid k-1}+B_{k-1} u_{k-1}$

Predicted estimate covariance: $P_{k \mid k-1}=F_{k} P_{k-1 \mid k-1} F_{k}^{T}+Q_{k-1}$

B. Measurement Update

Innovation (residual) covariance: $S_{k}=H_{k} P_{k \mid k-1} H_{k}^{T}+R_{k}$ 
Optimal Kalman gain: $K_{k}=P_{k \mid k-1} H_{k}^{T} S_{k}^{-1}$

Updated State estimate: $\hat{X}_{k \mid k}=\hat{X}_{k \mid k-1}+K_{k}\left(Z_{k}-H_{k} \hat{X}_{k \mid k-1}\right)$

Updated estimate covariance: $P_{k \mid k}=\left(I-K_{k} H_{k}\right) P_{k \mid k-1}$

Where:

$\hat{X}_{k \mid k}$ is the estimate of state at time $k$ given observations up to and including time $k$.

$K$ is the Kalman gain

$H$ is the measurement model

$Z_{k}$ is the measurement at time $k$

$F$ is state transition matrix

$B$ is input matrix

$P$ is estimate covariance

$R$ is measurement covariance

$Q$ is process covariance.

In this application, Kalman filter is implemented for the state variables with linear relationships ie Position and Velocity. Below are some mathematical computations for the three-dimensional position and velocity for this design:

$\mathrm{x}=\left[P^{T}, V^{T}\right]^{T}$

$$
\begin{aligned}
\text { where } \mathrm{P} & =\left[P_{x}, P_{y}, P_{z}\right]^{T} \quad \text { is the position vector } \\
\mathrm{v} & =\left[v_{x}, v_{y}, v_{z}\right]^{T} \quad \text { is the velocity vector }
\end{aligned}
$$

whose elements are defined in the $\mathrm{x}, \mathrm{y}, \mathrm{z}$ axes

Equations of motion, generating the dynamic equations of the process model are given as:

$$
\begin{aligned}
& x_{k}=\left[\begin{array}{l}
P_{k} \\
v_{k}
\end{array}\right]=\left[\begin{array}{c}
\left.P_{k-1}+\begin{array}{r}
v_{k-1} \Delta t+\frac{1}{2} a_{k-1} \Delta t^{2} \\
v_{k-1}+
\end{array}\right] a_{k-1} \Delta t
\end{array}\right] \\
& x_{k}=P_{x}=x_{k-1}+\dot{x} \Delta T+a_{x} 1 / 2 \Delta T^{2} \\
& P_{y}=y_{k-1}+\dot{y} \Delta T+a_{y} 1 / 2 \Delta T^{2} \\
& P_{z}=z_{k-1}+\dot{z} \Delta T+a_{z} 1 / 2 \Delta T^{2} \\
& V_{x}=\dot{x}+a_{x} \Delta T \\
& V_{y}=\dot{y}+a_{y} \Delta T \\
& V_{z}=\dot{z}+a_{z} \Delta T
\end{aligned}
$$

$$
x_{k}=\left[\begin{array}{cccccc}
1 & 0 & 0 & \Delta T & 0 & 0 \\
0 & 1 & 0 & 0 & \Delta T & 0 \\
0 & 0 & 1 & 0 & 0 & \Delta T \\
0 & 0 & 0 & 1 & 0 & 0 \\
0 & 0 & 0 & 0 & 1 & 0 \\
0 & 0 & 0 & 0 & 0 & 1
\end{array}\right]\left[\begin{array}{c}
x \\
y \\
z \\
\dot{x} \\
\dot{y} \\
\dot{z}
\end{array}\right]+\left[\begin{array}{ccc}
1 / 2 \Delta T^{2} & 0 & 0 \\
0 & 1 / 2 \Delta T^{2} & 0 \\
0 & 0 & 1 / 2 \Delta T^{2} \\
\Delta T & 0 & 0 \\
0 & \Delta T & 0 \\
0 & 0 & \Delta T
\end{array}\right]\left[\begin{array}{l}
a_{x} \\
a_{y} \\
a_{z}
\end{array}\right]
$$


$x_{k}=\left[\begin{array}{cc}I_{3 X 3} & I_{3 X 3} \Delta T \\ 0_{3 X 3} & I_{3 X 3}\end{array}\right] x_{k-1}+\left[\begin{array}{c}\frac{1}{2} I_{3 X 3} \Delta T^{2} \\ I_{3 X 3} \Delta T\end{array}\right] a_{k-1}$

$a_{k-1}=\tilde{a}_{k-1}+e_{k-1}$

$e_{k-1} \sim \mathrm{N}\left(0, I_{3 X 3} \sigma_{e}^{2}\right)$.

$e_{k-1}$ denotes the noise of the accelerometer output.

Process covariance, $\mathbf{Q}=\left[\begin{array}{c}1 / 2 I_{3 X 3} \Delta T^{2} \\ I_{3 \times 3} \Delta T\end{array}\right] I_{3 X 3} \sigma_{e}^{2}\left[\begin{array}{c}1 / 2 I_{3 X 3} \Delta T^{2} \\ I_{3 X 3} \Delta T\end{array}\right]$

$=\left[\begin{array}{cc}\frac{1}{4} I_{3 X 3} \Delta T^{4} & 0_{3 X 3} \\ 0_{3 X 3} & I_{3 X 3} \Delta T^{2}\end{array}\right] \sigma_{e}^{2}$

Recall, Process model/ Time update:

$x_{k}=\mathrm{F} x_{k-1}+\mathrm{B} u_{k-1}+w_{k-1}$

Where:

$\mathrm{F}=\left[\begin{array}{cc}I_{3 X 3} & I_{3 \times 3} \Delta T \\ 0_{3 \times 3} & I_{3 \times 3}\end{array}\right]$

$\mathrm{B}=\left[\begin{array}{c}\frac{1}{2} I_{3 \times 3} \Delta T^{2} \\ I_{3 \times 3} \Delta T\end{array}\right]$

$w_{k-1} \sim \mathrm{N}(0, \mathrm{Q})$

The GPS receiver provides position and velocity measurements corrupted by measurement noise $v_{k}$ as:

$z_{k}=\left[\begin{array}{l}P_{K} \\ V_{K}\end{array}\right]+v_{k}$

Deriving the measurement model becomes straight forward, given as:

$z_{k}=\mathrm{H} x_{k}+v_{k}$

Where $\mathrm{H}=I_{6 \times 6}$

$v_{k}=\mathrm{N}(0, \mathrm{R})$

$\mathbf{Q}$ and $\mathbf{R}$ are constant for every time step

$\mathrm{R}=\left[\begin{array}{cc}I_{3 X 3} \sigma_{p}^{2} & 0_{3 X 3} \\ 0_{3 X 3} & I_{3 X 3} \sigma_{v}^{2}\end{array}\right]$

$\sigma_{p}^{2}$ - noise variance for GPS position

$\sigma_{v}^{2}$ - noise variance for GPS velocity 


\subsection{Linearization (Extended Kalman filter)}

The discrete Kalman filter is applicable to linear systems, but most real-world problems are usually non-linear, either in its process or measurement model. In the integration of GPS and Kalman filter for instance the attitude is a non-linear parameter. In the EKF (extended Kalman filter), non-linear state and measurement equations is represented as:

$\dot{X}(t)=f(X(t), U(t))+w(t)$

$Z_{m}(t)=h(X(t), U(t))+v(t)$

Where:

f is the non-linear state equation

$\mathbf{h}$ is the non-linear measurement equation

$\mathbf{U}$ is the input vector

$\mathbf{w}$ and $\mathbf{v}$ represent process and measurement model noise.

Linearization of the above state and measurement nonlinear equations are done about the prior estimate of the state and each instant of time, by calculating the Jacobian with respect to the state variables as:

$$
F_{k}=\frac{\partial f}{\partial X}(\hat{X}(k), U(k)) \text { and } H_{k}=\frac{\partial h}{\partial X}(\hat{X}(k), U(k))
$$

And for discrete time implementation of EKF, the linearized system is discretized in time by computing the state transition matrix from $F_{k}$ where $\Delta \mathrm{t}$ is time interval [17]

$$
\Phi_{k \mid k-1}=e^{F_{k} \Delta t} \cong\left[I+F_{k} \Delta t\right]
$$

The time and measurement update are given below:

\section{A. Time update}

$\hat{X}_{k \mid k-1}=\hat{X}_{k-1 \mid k-1}+\int_{k-1}^{k} f(X(t), U(t)) d t$
$\mathrm{P}_{k \mid k-1}=\Phi_{k \mid k-1} \mathrm{P}_{k \mid k-1} \Phi_{k \mid k-1}^{T}$

\section{B. Measurement update}

$S_{k}=H_{k} P_{k \mid k-1} H_{k}^{T}+R_{k}$

$K_{k}=P_{k \mid k-1} H_{k}^{T} S_{k}^{-1}$

$\hat{X}_{k \mid k}=\hat{X}_{k \mid k-1}+K_{k}\left(Z_{k}-h_{k}\left(\hat{X}_{k \mid k-1}\right)\right)$

$P_{k \mid k}=\left(I-K_{k} H_{k}\right) P_{k \mid k-1}$

\section{Machine/Deep learning}

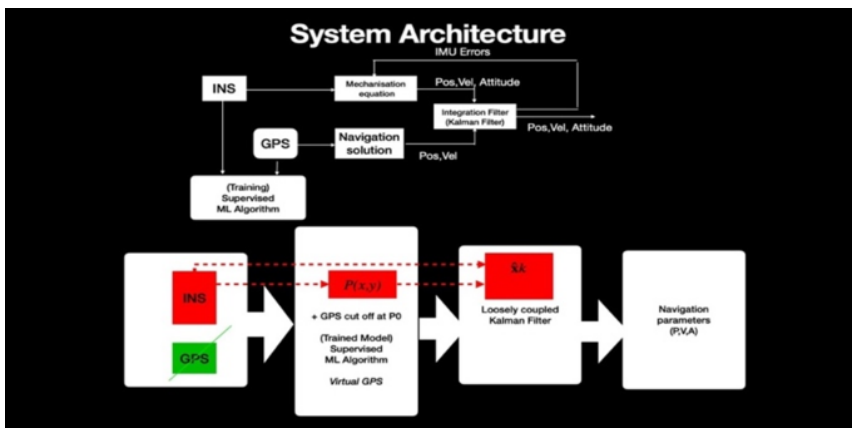

Figure 2: Proposed system architecture using combined GPS and INS 
As discussed earlier, this research work's approach is to solving the inherent shortcomings in sensor fusion of the GPS/INS using Kalman filter by integrating a machine learning algorithm to improve overall performance. The principal strategy of the proposed neural network and Kalman filter hybrid approach is to map the relationship between vehicle manoeuvre and INS positioning error during each Kalman filter update with Neural network. The degradation of the system can be offset if the INS positioning error can be estimated. NN training is carried out at GPS sampling rate, such that when GPS is unavailable, the well trained NN can be used to provide measurement update.

The first stage of its functioning is the training phase and the final phase is the prediction mode

\section{System during training phase:}

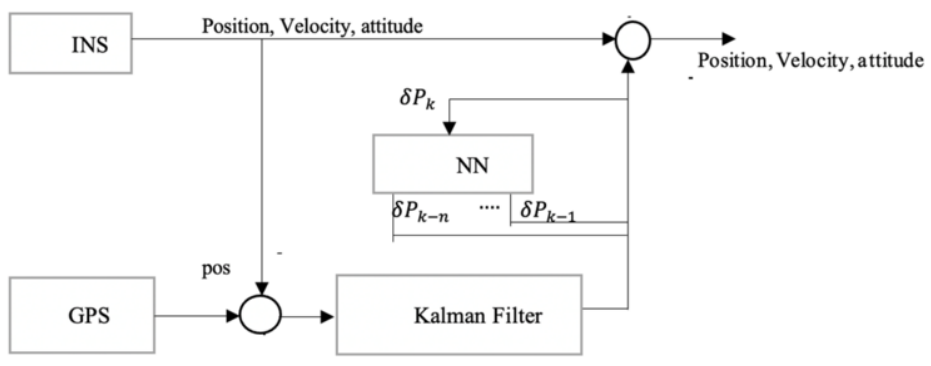

Figure 3: Neural Network training mode CAD-model

\section{System during NN prediction phase:}

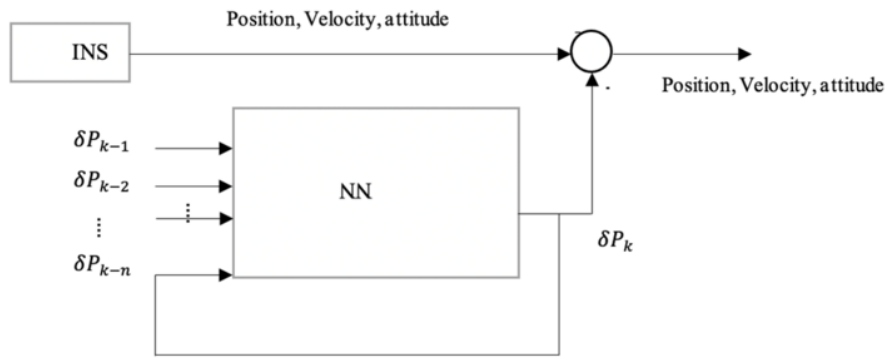

Figure 4: Neural network prediction mode CAD-model

Neural network training input and output selection:

Selecting proper inputs and outputs to the Neural network is key to proper training. The outputs to the $\mathrm{NN}$ or training targets $\delta P_{k}$ are selected to be the positioning error with respect to GPS, which corresponds to the measurement of the Kalman filter.

$$
N N_{\text {out }}=\delta P_{k}=\left[\delta P_{N}, \delta P_{E}, \delta P_{D}\right.
$$


The input to the NN would be represented as the variation or changes in velocity and attitude/orientation at each epoch.

$$
N N_{I N}=\delta P_{k-n}=[\Delta v, \Delta \phi]
$$

\section{Experimental Work}

The initial experimental work carried out in MATLAB showed the first phase result of this research programme:

based on the mathematical model of the INS/GPS integrated navigation system and utilization of the north east Up coordinates, we exploited the extended Kalman filtering to estimate system states. GPS is set at sampling rate: $10 \mathrm{~Hz}$, while IMU is set at $100 \mathrm{~Hz}$. In a typical system, the IMU components (accelerometer and gyroscope) run at relatively high sample rate, conversely the GPS runs at a low sample rate. The entire simulation time taken 30seconds.

Vehicle moves horizontally along the equatorial North direction, towards the east and its trajectory if continued would return back to the local origin. simulation parameters are set as follows: 1) GPS initial position is located at 42.2825 degrees North latitude and -71.343 degrees East longitude, 2) the height is set to $53.0352 \mathrm{~m}, 3$ ) the initial rate is set to $2.50 \mathrm{~m} / \mathrm{s}$. 4). GPS decay factor is set at 0.55 ) Horizontal and Vertical position accuracy is set at 1.06 ) GPS velocity accuracy is set at 0.1. 7) End-to-End Simulation Position RMS Error X: 1.16, Y: 0.99, Z: 0.03, all in meters. 8) End-to-End Quaternion Distance RMS Error: 0.09 (degrees). The ground vehicle has two velocity constraints that assume it does not bounce off the ground or slide on the ground. These constraints are applied using the extended Kalman filter update equations. These updates are applied to the filter states at a rate of IMU-Sample-Rate / Decimation-Factor Hz.

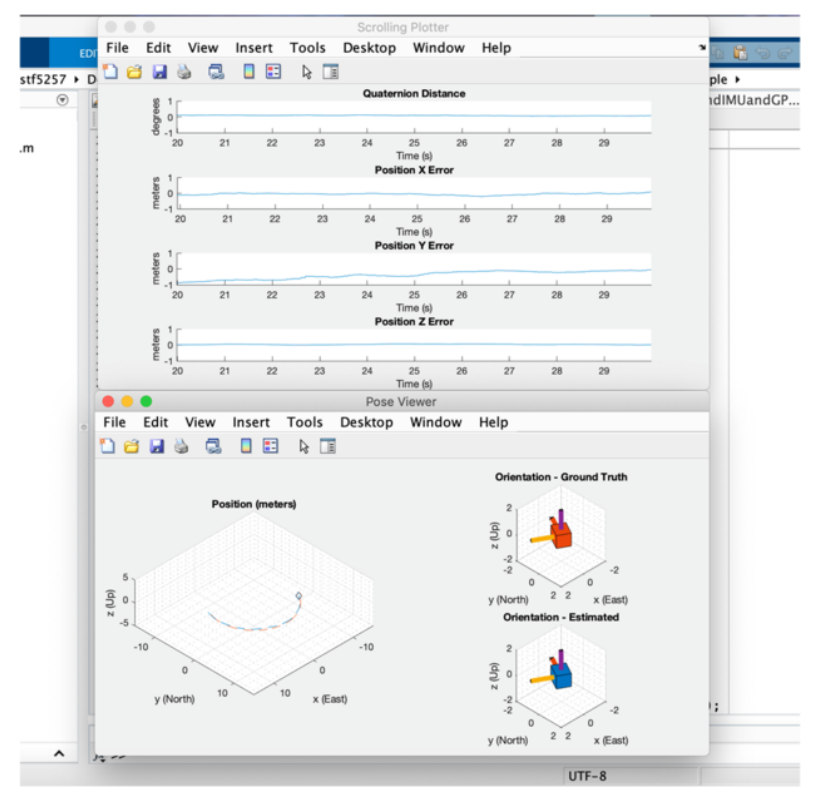

Figure 5: Showing Experimental work 
The simulation process is implemented by MATLAB, and then the extended Kalman filter is utilized to test the performance of filter divergence suppression and the wide range of adaptive capacity. Observe the error along the $\mathrm{z}$ (up) axis remain relatively unchanged and its values nearer to 0 , as there isn't really movement along the axis. Furthermore, the estimated error in the $\mathrm{x}$ (East) and $\mathrm{y}$ (North) positions, grow and thus estimates and ground truth values diverge, but as the filter begins to settle in at about $t=$ $20 \mathrm{~s}$, the error in $\mathrm{x}$ and $\mathrm{y}$ positions begins to approach 0 . First in position $\mathrm{x}$ and then at about $\mathrm{t}=25 \mathrm{~s}$ in position $\mathrm{y}$. ultimately the Kalman filter provides optimized state solution for INS/ GPS integration, however the short comings of it, is overcome when its hybridized with a neural network, discussed subsequently in this project

\section{Conclusion/ future work}

This paper focused on machine learning solutions to offset the accuracy deviations of a GPS/IMU based Localization system. It details the working of an extended Kalman filter, used to fuse both GPS and IMU parameters, to give an optimal solution for vehicle localization; the experiments showed converging values of localization parameters to optimal POSE values, as it's iterated over time. The next steps of this ongoing research program would include a well-trained neural network to predict and compensate the interrupted signal position error. A multi-layer $\mathrm{NN}$ is trained to map the vehicle maneuver with INS prediction errors during each GPS epoch, which is the input of the EKF. During GPS signal blockages, the NN can be used to predict the INS errors for EKF measurement updates, and in this way to improve navigation solutions. Future research in employment of cutting-edge technology to accurate vehicle localization and tracking can consider the deployment of available 4G/5G infrastructures as beacons; triangulating these beacons for position determination while reinforcing its signals when it suffers degradation using machine learning algorithms as described in this work.

\section{References}

[1] Reid, T., Houts, S., Cammarata, R., Mills, G., Agarwal, S., Vora, A. and Pandey, G.,. Localization Requirements for Autonomous Vehicles. SAE International Journal of Connected and Automated Vehicles, 2(3).. 2019

[2] I. Skog and P. Händel, "In-Car Positioning and Navigation Technologies- A Survey," IEEE Transactions on Intelligent Transportation Systems, vol. 10, no. 1, pp. 4-21, 2009.

[3] F. Zhang, H. Stähle, G. Chen, C. C. C. Simon, C. Buckl, and A. Knoll, "A sensor fusion approach for localization with cumulative error elimina- tion," in 2012 IEEE International Conference on Multisensor Fusion and Integration for Intelligent Systems (MFI). IEEE, 2012, pp. 1-6.

[4] Chistyakov, V., Mikhailov, N., Pospelov, S., Vasil'ev, M. and Vasil'eva, N., . Method of fast first fix for low cost GNSS receivers. Gyroscopy and Navigation, 2(2), pp.63-68. (2011)

[5] A. Brown, and Y. Lu, "Performance Test Results on an Integrated GPS/MEMS Inertial Navigation Package," in Proc. Institute of Navigation GNSS 2004, Long Beach, CA, Sept. 2004, pp. 825-832.

[6] Goodall, C., El-Sheimy, N. and Chiang, K.-W., The development of a GPS/MEMS INS integrated system utilizing a hybrid processing architecture, ION GNSS 18th International Technical Meeting of the Satellite Division, Long Beach, CA, pp. 1444-1455. 2005

[7] Martin Dekan, Ladislav Jurišica, Anton Vitko František Duchoň, " Some Applications of Laser Rangefinder in Mobile Robotics," Control Engineering and Applied Informatics vol. 14, no. 2, pp. 5057.2012 